\title{
Blind Subband Beamforming With Time-Delay Constraints for Moving Source Speech Enhancement
}

\author{
Zohra Yermeche, Member, IEEE, Nedelko Grbić, Member, IEEE, and Ingvar Claesson, Member, IEEE
}

\begin{abstract}
A new robust microphone array method to enhance speech signals generated by a moving person in a noisy environment is presented. This blind approach is based on a two-stage scheme. First, a subband time-delay estimation method is used to localize the dominant speech source. The second stage involves speech enhancement, based on the acquired spatial information, by means of a soft-constrained subband beamformer. The novelty of the proposed method involves considering the spatial spreading of the sound source as equivalent to a time-delay spreading, thus, allowing for the estimated intersensor time-delays to be directly used in the beamforming operations. In comparison to previous approaches, this new method requires no special array geometry, knowledge of the array manifold, or acquisition of calibration data to adapt the array weights. Furthermore, such a scheme allows for the beamformer to efficiently adapt to speaker movement. The robustness of the time-delay estimation of speech signals in high noise levels is improved by making use of the non-Gaussian nature of speech trough a subband Kurtosis-weighted structure. Evaluation in a real environment with a moving speaker shows promising results, with suppression levels of up to $16 \mathrm{~dB}$ for background noise and interfering (speech) signals, associated to a relatively small effect of speech distortion.
\end{abstract}

Index Terms-Array signal processing, delay estimation, speech enhancement.

\section{INTRODUCTION}

A S SPEECH processing technology matures and telecommunication becomes more prevalent, a new generation of speech acquisition applications emerges in which voice connectivity extends to personal computers through Internet telephony, audio conferencing, voice control, and speech recognition [1]. These applications involve the objective to enable natural and accurate communication for speakers at remote distances from acquisition devices, and in adverse noise environments. Therefore, the problem at hand consists of extracting a desired speech signal from the captured noisy sound field. Successful speech enhancement solutions are expected to achieve efficient noise and interference reduction, speech dereverberation in highly reverberant enclosures, and, for mobile environments, the provision of a capability to adapt to speaker movement [1], [2]. Ultimately, it is desirable to solve the aforementioned problem blindly, i.e., without prerequisite knowledge about the speaker or the acoustical environment. Methods using microphone arrays aim to address the problem in its totality. A large diversity of blind array processing algorithms derived from classical

Manuscript received September 12, 2006; revised May 2, 2007. The associate editor coordinating the review of this manuscript and approving it for publication was Dr. Alex Acero.

The authors are with the Department of Signal Processing, Blekinge Institute of Technology, SE-372 25 Ronneby, Sweden (e-mail: zye@bth.se).

Digital Object Identifier 10.1109/TASL.2007.903309 signal processing methods can be found in literature. Among them, blind signal separation (BSS) based on independent component analysis (ICA) prominently stands out [3]-[10]. It aims to separate unknown sources from observed mixtures based solely on their statistical independence [11], [12]. Regrettably, most BSS algorithms display an inherent limitation with regard to separation capacity in reverberant environments. To overcome the multipath (i.e., reverberant) environment restrictions, a derivation of the ICA-algorithm to include the deconvolution of mixtures is presented in the time domain [3] and in the frequency domain [4]. A subband approach for BSS was, however, proven to accomplish much better performance in highly reverberant enclosures [5]. Furthermore, to remove the remaining interferences in the ICA-separated signals, different postprocessors were suggested. For instance, postprocessing based on adaptive noise cancellation [7], spectral substraction [8], time-frequency masking [9], and more recently, direction-of-arrival (DOA) estimation-based beamforming [10] were suggested to further enhance the separated speech signals.

Another criteria for blind array processing is the Kurtosis maximization [13], [14]. The Kurtosis is a measure based on fourth- and second-order statistics, and it is commonly used as a quantitative measure of non-Gaussianity. The goal here is to extract signals of a certain class of distributions and exploit the a priori knowledge that the signal to be recovered is speech. The idea behind maximizing the Kurtosis of the received signals stems from the fact that speech is a super-Gaussian signal and has a positive Kurtosis, while background noise most commonly displays a nearly Gaussian distribution with a normalized Kurtosis value close to zero. However, most of the blind array processing methods mentioned above rely on constantly present signals to converge, as opposed to the methods described in the sequent.

Another inherent characteristic of speech, consequent to its non-Gaussianity, is its sparseness in the time-frequency domain [15]. An increasingly popular approach to separate simultaneously active speech signals is based on time-frequency masking [9], [15]. Such methods are, however, sensitive to high noise levels and not suitable for reverberant environments. In fact, in low signal-to-noise ratio (SNR) scenarios, the noise is more likely to contaminate (and cover) the time-frequency points with speech energy. Similarly, reverberation makes it difficult to distinguish time-frequency points associated with different sources.

Concurrently, the inherent ability of microphone arrays to exploit the spatial correlation of the multiple received signals has enabled the development of combined temporal and spatial filtering algorithms known as beamforming techniques [2]. In these methods, a beam is created towards a target direction, so 
as to maintain its gain and phase, while spatial nulls are created towards jammer directions. Among the many beamforming techniques, some employ optimal filtering [16], [17], signal subspace concepts [18], or spectral subtraction [19]. Many of these algorithms rely on voice activity detection (VAD). This is needed in order to avoid source signal cancellation effects, which may result in unacceptable levels of speech distortion [2]. Methods based on calibration data have been developed to circumvent the need of a VAD [17], [20], [21]. While calibration-based beamformers present the benefit of taking into consideration the real room acoustical properties, these methods require access to new calibration data whenever the acoustical properties change. This can occur due to changes in the array manifold, source location, microphone aging, etc. Another approach presented in [16], [22], and [23], relies on free-field assumptions to obtain the desired source covariance function. This approach is based on a propagation model and a known target source location rather than formed from calibration data. While such approach relies on the availability of a good estimate of the source location, it increases the probability for errors due to the manifold model and microphone mismatch.

This paper presents a new robust blind speech enhancement algorithm. The proposed structure consists of a subband soft-constrained adaptive beamformer combined with a subband weighted time-delay estimation (TDE) method. First, a new robust method for TDE is proposed to estimate the propagation time difference of arrival (TDOA) of a dominant speech source received by a sensor array. This method is based on the steered response power with phase transform (SRP-PHAT) algorithm, modified to work in a subband structure. A Kurtosis measure is used in the subband domain to evaluate the speech content of the subband signals and to weight their contribution in the TDE. This approach is related to the higher order statistics (HOS)-based VAD, such as presented in [24] and [25]. It is used to improve the TDE performance in high-noise environments. The estimated time-delays are used directly in the recursive update of the beamformer's weight vector. A subband recursive least squares (RLS)-based soft-constrained beamformer described in [21] is employed. To secure the spatial-temporal passage of the desired source signal, a soft-constraint is formed on the weight update from the acquired source TDE and a propagation model. The source model assumes spatially spread speech sources. In fact, a time-delay spread model centered on the estimated source TDOA is used to represent the spatial spreading of the desired source as well as to include errors in the TDE. This representation has the advantage of being independent of the array constellation; hence, it does not require any a priori knowledge of a manifold model or prior acquisition of calibration data. Another benefit is that it takes into consideration the nonideal nature of microphones and other discrepancies inherent to the use of an acoustical environment model. Such a scheme also allows for an efficient adaptation of the beamformer to speaker movement. It should be noted, however, that a requirement on a maximum intersensor distance is imposed to prevent spatial aliasing effects. In addition to substantially reducing the computational complexity of the beamformer by using a subband scheme, this structure allows for a frequency-dependent constraint design. The objective is to take into consideration the fact that small time-delay changes in low-frequency bands correspond to large spatial openings of the beamformer. The designed constraint is therefore adjusted in each subband correspondingly.

The remainder of the paper is organized as follows. In Section II, a general signal model for sound propagation is given in the time-frequency domain. Section III offers a detailed presentation of the existing constrained subband beamforming approach, with its two variants: the calibration-based and the source-spread-model-based algorithms. The proposed delay-based constrained subband beamformer is described in Section IV. Section V gives an evaluation of this method and a comparison to the existing algorithms mentioned above. Finally, Section VI concludes the paper.

\section{SignAl Modeling}

Let us consider a noisy acoustical environment where a desired source signal $s(t)$ at time instant $t$ is impinging on an array of $M$ microphones. The sound field captured by the array's $m$ th sensor, $m=1,2, \ldots, M$

$$
x_{m}(t)=x_{s, m}(t)+x_{i, m}(t)+x_{n, m}(t)
$$

consists of the propagating signal $s(t)$ to the $m$ th sensor $x_{s, m}(t)$ and also includes contributions from interference and background noise, $x_{i, m}(t)$ and $x_{n, m}(t)$, respectively.

In the particular case of a point source signal, $s(t)$, we have $x_{s, m}(t)=s(t) * h_{s, m}(t)$, where $h_{s, m}(t)$ is the corresponding impulse response and $(*)$ represents the convolution operator. Subband frequency transformation is one way to conveniently replace the time-domain convolution by a multiplication in the frequency-domain. From here onwards, a time-frequency representation of the signals obtained through spectral decomposition is employed [26]. Since the processing of narrow-band signals requires lower sample rates, the frequency transform is followed by a decimation operation [26]. The signal $s(t)$ is sampled and decomposed into a set of $K$ narrow-band signals $S(k, l)$, where $k=0,1, \ldots, K-1$ is the subband index, and $l$ is the sample index after decimation. The index $k$ refers to the frequency subband centered at the angular frequency $\Omega_{k}=2 \pi F_{s} k / K$, where $F_{s}$ is the sampling frequency. Hence, the received source input vector is expressed in subband $k$ as

$$
\mathbf{X}_{s}(k, l)=S(k, l) \mathbf{H}\left(k, R_{s}, \theta_{s}\right)
$$

where

$$
\begin{aligned}
\mathbf{X}_{s}(k, l) & =\left[X_{s, 1}(k, l), \ldots, X_{s, M}(k, l)\right]^{T} \\
\mathbf{H}\left(k, R_{s}, \theta_{s}\right) & =\left[H_{1}\left(k, R_{s}, \theta_{s}\right), \ldots, H_{M}\left(k, R_{s}, \theta_{s}\right)\right]^{T}
\end{aligned}
$$

$X_{s, m}(k, l)$ is the subband representation of $x_{s, m}(t)$, $H_{m}\left(k, R_{s}, \theta_{s}\right)$ is the frequency response of $h_{s, m}(t)$ for subband $k$, assumed to be invariant, and $\left(R_{s}, \theta_{s}\right)$ is the radius and angle of the source, $s(t)$, with respect to a reference point. The symbol $(\cdot)^{T}$ stands for transpose. The complete received signal may correspondingly be expressed by

$$
\mathbf{X}(k, l)=\mathbf{X}_{s}(k, l)+\mathbf{X}_{i}(k, l)+\mathbf{X}_{n}(k, l)
$$


where $\mathbf{X}_{i}(k, l)$ and $\mathbf{X}_{n}(k, l)$ are the received interference and noise input vectors in the time-frequency domain, respectively. The frequency response vector $\mathbf{H}\left(k, R_{s}, \theta_{s}\right)$ can be decomposed into its direct-path and multipath reverberation vector components, $\mathbf{H}\left(k, R_{s}, \theta_{s}\right)=\mathbf{H}_{d}\left(k, R_{s}, \theta_{s}\right)+\mathbf{H}_{r}\left(k, R_{s}, \theta_{s}\right)$. In fact, the frequency response of the direct-path from the source to the array $\mathbf{H}_{d}\left(k, R_{s}, \theta_{s}\right)$, referred to as the source steering vector, can be modeled as

$$
\begin{aligned}
\mathbf{H}_{d}\left(k, R_{s}, \theta_{s}\right)=\left[a_{1}\left(R_{s}, \theta_{s}\right) e^{-j \Omega_{k} \tau_{1}\left(R_{s}, \theta_{s}\right)}, \ldots,\right. \\
\left.a_{M}\left(R_{s}, \theta_{s}\right) e^{-j \Omega_{k} \tau_{M}\left(R_{s}, \theta_{s}\right)}\right]^{T} .
\end{aligned}
$$

This formulation implies that the direct-path input signal to the $m$ th sensor is a delayed and scaled version of the source signal, with a delay $\tau_{m}\left(R_{s}, \theta_{s}\right)$ and an attenuation factor $a_{m}\left(R_{s}, \theta_{s}\right)$.

However, typical speech sources, such as a human mouth or a hands-free loudspeaker, are regarded as spatially spread sources. A spatially spread source can be modeled as an infinite number of stationary and independent point sources, clustered closely within a small area in space, $\vartheta_{s}$

$$
S(k, l)=\iint_{\vartheta_{s}} S_{p}(k, l, R, \theta) d R d \theta
$$

where $S_{p}(k, l, R, \theta)$ is the subband representation of the signal component at position $(R, \theta)$. Hence, the received microphone input vector generated by the speech signal is expressed as

$$
\begin{aligned}
\mathbf{X}_{s}(k, l) & =\iint_{\vartheta_{s}} S_{p}(k, l, R, \theta) \mathbf{H}(k, R, \theta) d R d \theta \\
& =\mathbf{X}_{s d}(k, l)+\mathbf{X}_{s r}(k, l)
\end{aligned}
$$

with

$$
\begin{aligned}
& \mathbf{X}_{s d}(k, l)=\iint_{\vartheta_{s}} S_{p}(k, l, R, \theta) \mathbf{H}_{d}(k, R, \theta) d R d \theta \\
& \mathbf{X}_{s r}(k, l)=\iint_{\vartheta_{s}} S_{p}(k, l, R, \theta) \mathbf{H}_{r}(k, R, \theta) d R d \theta
\end{aligned}
$$

where we refer to the component of the signal vector $\mathbf{X}_{s}(k, l)$ for the direct-path as $\mathbf{X}_{s d}(k, l)$ and for the multipath reverberations as $\mathbf{X}_{s r}(k, l)$. Let us take a closer look at the direct-path signal received at the array's $m$ th sensor, $m=1,2, \ldots, M$

$X_{s d, m}(k, l)=\iint_{\vartheta_{s}} S_{p}(k, l, R, \theta) a_{m}(R, \theta) e^{-j \Omega_{k} \tau_{m}(R, \theta)} d R d \theta$

where $\tau_{m}(R, \theta)$ and $a_{m}(R, \theta)$ are the delay parameter and attenuation factor of the direct-path from position $(R, \theta)$ to the $m$ th sensor. For a homogeneously spread source with closely clustered points, the following assumption can be made: $a_{m}(R, \theta)=\tilde{a}_{s, m}$, for all points $(R, \theta)$ included in $\vartheta_{s}$. Furthermore, we assume a homogeneous source signal power $A_{s}$ received at all elements in the array ${ }^{1}$ (i.e., $\tilde{a}_{s, m}=A_{s}$,

\footnotetext{
${ }^{1}$ The intermicrophone spacing is small in comparison to the distance between the array and the source outside of the extreme near-field. The discrepancies in power received by the array elements is thus negligible.
}

for all $m$ ). Hence, using the general model for a spatially spread source given in (8), the source covariance matrix can be estimated from the direct-path signal as

$$
\begin{aligned}
\mathbf{R}_{s}(k) & =E\left\{\mathbf{X}_{s d}(k, l) \mathbf{X}_{s d}(k, l)^{H}\right\} \\
& \approx P_{s}(k) \iint_{\vartheta_{s}} e^{-j \Omega_{k} \boldsymbol{\tau}(R, \theta)} e^{-j \Omega_{k} \boldsymbol{\tau}(R, \theta)^{H}} d R d \theta
\end{aligned}
$$

where $P_{s}(k)=A_{s}^{2} E\left\{S(k, l) S(k, l)^{*}\right\}$ is the source power spectral density (PSD) for subband $k$, and $\boldsymbol{\tau}(R, \theta)=$ $\left[\tau_{1}(R, \theta), \tau_{2}(R, \theta), \ldots, \tau_{M}(R, \theta)\right]^{T}$ is the time-delay vector of the direct-path from the point source $S_{p}(k, l, R, \theta)$ to each element in the array. Here, the symbol $E\{\cdot\}$ denotes the statistical expectation, the notation $(\cdot)^{H}$ stands for the Hermitian transpose, and $(\cdot)^{*}$ is the complex conjugate. One way to simplify the formulation in (9) is to consider the vector $\boldsymbol{\tau}(R, \theta)$ as the TDOA of the source signal to the sensor-array in relation to the signal received by the reference microphone with index $c$. Thus, without loss of generality, we take $c=1$ and $\boldsymbol{\tau}(R, \theta)=\left[0, \tau_{1,2}(R, \theta), \ldots, \tau_{1, M}(R, \theta)\right]^{T}$, where $\tau_{1, m}(R, \theta)=\tau_{m}(R, \theta)-\tau_{1}(R, \theta)$ for $m=2,3, \ldots, M$.

\section{Subband Constrained AdAPtive BEAmforming}

\section{A. Optimal and Adaptive Beamforming}

The output of the beamformer for subband $k$ and time-index $l$ is given by

$$
Y(k, l)=\mathbf{W}(k)^{H} \mathbf{X}(k, l)
$$

where $\mathbf{W}(k)$ is the $M \times 1$ beamformer's subband weight vector. For speech enhancement applications, the beamformer optimizes the array output so as to minimize contributions from noise and interference signals. The optimal weight vector required to minimize the expected square difference between the output and the desired signal $S(k, l)$

$$
\mathbf{W}_{\text {opt }}(k)=\arg \min _{\mathbf{W}(k)} E\left\{|Y(k, l)-S(k, l)|^{2}\right\}
$$

is obtained by the standard Wiener solution following [27]

$$
\mathbf{W}_{\text {opt }}(k)=\mathbf{R}_{\mathbf{x}}(k)^{-1} \mathbf{r}_{\mathbf{x} s}(k)
$$

where

$$
\begin{aligned}
& \mathbf{R}_{\mathbf{x}}(k)=E\left\{\mathbf{X}(k, l) \mathbf{X}(k, l)^{H}\right\} \\
& \mathbf{r}_{\mathbf{x} s}(k)=E\left\{\mathbf{X}(k, l) S(k, l)^{*}\right\}
\end{aligned}
$$

are the input covariance matrix and cross-covariance vector, respectively.

When operating in a nonstationary environment, the weights of the beamformer require updating in order to follow the statistical variations of the observable data. It should be noted that a nonmoving source signal is spatially stationary, as a fixed location will always excite the same correlation patterns between microphones. Hence, the corresponding spatial source statistics are constant. Consequently, the adaptive algorithm, in the case of a spatially stationary source, can be reduced to a time-varying filter tracking the behavior of the noise in order to suppress it. 
In practice, the weighted deterministic data covariance matrix calculated from the observed data as follows:

$$
\hat{\mathbf{R}}_{x}(k, l)=\sum_{p=0}^{l} \lambda^{l-p+1} \mathbf{X}(k, p) \mathbf{X}(k, p)^{H}
$$

is used instead of (13). The parameter $\lambda$ is a forgetting factor with the purpose of allowing for tracking variations in the surrounding noise environment.

\section{B. Constrained Beamforming}

A direct application of the method described in the previous section results in distortion of the desired signal, mainly in highnoise environments. This limitation can be circumvented by the introduction of a soft-constraint on the adaptive filter weights, based on knowledge of the source position, so as to secure the passage of the desired signal. The weight vector for the constrained least squares (LS) minimization can be formulated as [21]

$$
\begin{aligned}
\mathbf{W}(k, l) & =\hat{\mathbf{R}}(k, l)^{-1} \mathbf{r}_{\mathbf{x} s}(k) \\
& =\left(\mathbf{R}_{s}(k)+\hat{\mathbf{R}}_{x}(k, l)\right)^{-1} \mathbf{r}_{\mathbf{x} s}(k)
\end{aligned}
$$

where $\mathbf{R}_{s}(k)$ contains the fixed spatial correlation patterns of the source, while the signal-dependent correlation patterns of the source due to speech nonstationarity are incorporated in $\hat{\mathbf{R}}_{x}(k, l)$. Since the source covariance matrix $\mathbf{R}_{s}(k)$ is always forced to match the cross-covariance vector $\mathbf{r}_{\mathbf{x} s}(k)$, the beamformer's mainlobe is maintained constant towards the direction of the desired source. Hence, $\mathbf{R}_{s}(k)$ constitutes a soft constraint, which acts as a spatial passband. Additionally, it moderates the weight vector fluctuations generated by speech pauses and forces full rank properties of the total matrix $\hat{\mathbf{R}}(k, l)$, when no noise, interference, or speech is present.

In comparison to hard-constrained beamforming techniques [such as the linearly constrained minimum variance (LCMV) beamformer], the soft-constrained beamformer exploits both spectral and spatial characteristics of the speech and the noise to extract the speech [28]. By using a soft-constraint, the noise reduction can be performed over the complete space, hence, allowing for some speech distortion. On the other hand, a hard constraint would leave the speech subspace undistorted but would restrict the noise reduction to the remaining subspaces. Hence, as analytically formulated in [28], a tradeoff between levels of speech distortion and noise reduction can be achieved by choosing different constraints.

\section{Constrained Weighted RLS Beamformers}

A recursive formulation for the update of the beamforming weight vector is given in the Appendix. The recursive solution of the LS minimization problem given in (16) is based on a prerequisite knowledge of the source statistics $\mathbf{R}_{s}(k)$ and $\mathbf{r}_{\mathbf{x} s}(k)$. These statistics can be estimated, either based on some knowledge about the source or blindly. In various hands-free communication applications, such as a car scenario, the source position (in this case the location of the driver's head relative to the microphone array) is almost fixed and known approximately. Hence, source statistics can be estimated from data sequences

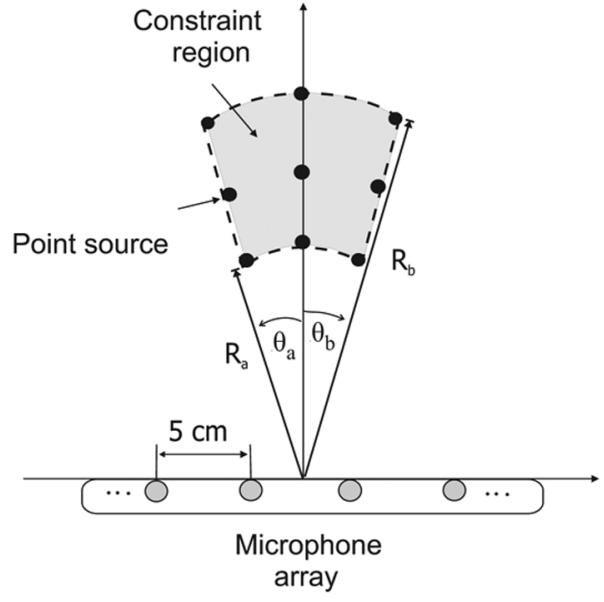

Fig. 1. Constraint region defined by the radii $\left[R_{a}, R_{b}\right]$ and the angles $\left[\theta_{a}, \theta_{b}\right]$, discretized here into (represented by) nine point sources.

uttered from the known source position and gathered by the sensor array in a calibration phase. The source statistics can also be calculated for the known source position, based on the signal models of (2) and (6). The major difference between these two approaches is in the desired signal chosen for the error minimization of (11), i.e., reverberant multipath source signal versus direct-path signal.

1) Calibration-Based Constrained Beamformer (CCWRLS): The calibrated constrained weighted RLS (CC-WRLS) beamformer was presented in [21]. The source covariance estimates are precalculated for a data set of $L$ block samples, gathered in a calibration phase, as follows:

$$
\begin{aligned}
\hat{\mathbf{R}}_{s}(k) & =\frac{1}{L} \sum_{l=0}^{L-1} \mathbf{X}_{s}(k, l) \mathbf{X}_{s}(k, l)^{H} \\
\hat{\mathbf{r}}_{s}(k) & =\frac{1}{L} \sum_{l=0}^{L-1} \mathbf{X}_{s}(k, l) X_{s, c}(k, l)^{*} .
\end{aligned}
$$

In this structure, the desired signal consists of the reverberant multipath source signal received at sensor with index $c$. The benefit is that the real room acoustical properties are taken into consideration, including errors due to microphone mismatch. Such an approach has been shown to be nearly optimal in signal-tonoise-plus-interference ratio (SNIR) sense [16].

2) Model-Based Constrained Beamformer (MBC-WRLS): The soft constraint is here defined for a specified region corresponding to the source location. The spatially spread source is modeled as an infinite number of short-time stationary and independent point sources clustered closely in space within a range of radii $\left[R_{a}, R_{b}\right]$ and inside the range of arrival angles $\left[\theta_{a}, \theta_{b}\right]$; see Fig. 1 . The spatial covariance matrix and covariance vector corresponding to the direct-path source signal vector are then given by [22]

$$
\begin{aligned}
\check{\mathbf{R}}_{s}(k) & =P_{s}(k) \int_{R_{a}}^{R_{b}} \int_{\theta_{a}}^{\theta_{b}} e^{-j \Omega_{k} \boldsymbol{\tau}(R, \theta)} e^{-j \Omega_{k} \boldsymbol{\tau}(R, \theta)^{H}} d R d \theta \\
\check{\mathbf{r}}_{s}(k) & =P_{s}(k) \int_{R_{a}}^{R_{b}} \int_{\theta_{a}}^{\theta_{b}} e^{-j \Omega_{k} \boldsymbol{\tau}(R, \theta)} d R d \theta .
\end{aligned}
$$


Small errors in the frequency response vector cause large radial errors in the corresponding source location for sources that are outside the extreme near-field [2]. Hence, an appropriate configuration involves defining the speech source area as a pie slice region, as depicted in Fig. 1. This configuration adequately contains the consequences of errors in the frequency response vector caused by the misplacement and gain variations of the microphones as well as by the error in the source position estimate. Additionally, a more convenient implementation can be achieved by using a discrete-space model of the speech source rather than the continuous-space model. By using a finite number $I$ of stationary and independent point sources clustered closely within the constraint area (see Fig. 1), the spatial covariance matrix and cross covariance vector of (19) and (20) can be approximated by

$$
\begin{aligned}
\check{\mathbf{R}}_{s}^{\prime}(k) & =P_{s}(k) \sum_{i=1}^{I} e^{-j \Omega_{k} \boldsymbol{T}\left(R_{i}, \theta_{i}\right)} e^{-j \Omega_{k} \boldsymbol{\tau}\left(R_{i}, \theta_{i}\right)^{H}} \Delta \vartheta_{i} \\
\check{\mathbf{r}}_{s}^{\prime}(k) & =P_{s}(k) \sum_{i=1}^{I} e^{-j \Omega_{k} \boldsymbol{\tau}\left(R_{i}, \theta_{i}\right)} \Delta \vartheta_{i}
\end{aligned}
$$

where $\left\{\Delta \vartheta_{i}\right\}$ covers the whole constraint region. The error introduced by this approximation can be made negligible by using a high enough number $I$ of point sources in the calculation. The calculated source statistics for a given source position are then exploited in the WRLS beamformer, resulting in the modelbased constrained WRLS (MBC-WRLS) beamformer.

\section{PRoposed Method}

For the CC-WRLS and MBC-WRLS beamformers described in the previous section, a prerequisite knowledge of the target source location is required. A TDOA method can be used to localize the source position and track it. One drawback of this approach is that it relies on a known array geometry. Thus, a small mismatch in the array manifold may result in large source location estimation errors. Furthermore, when the source is moving, a recalibration of the CC-WRLS beamformer and a recalculation of the source statistics for both methods is needed. This section describes the proposed blind subband beamforming algorithm for enhancing a dominant speech source in a noisy environment, referred to as the delay-based constrained WRLS blind (DBC-WRLSB) beamformer. First, a subband TDE method is described. The proposed structure is based on the SRP-PHAT algorithm defined for both the near-field and the far-field, where a subband approach is suggested to circumvent the limitation of such a TDE method in low SNR conditions. The advantage of performing the TDE in subbands is to exploit the nonflat frequency spectrum of the speech and its time-frequency sparseness to improve the quality of the delay estimates. In fact, the subbands with a higher SNR are most likely to give a more accurate time-delay estimate of the dominant speech source. Thus, the suggested structure includes a subband Kurtosis measure to weight the contribution of each subband signal in the TDE. Similarly, the properties of HOS of speech and noise have successfully been exploited in [24] and [25] to achieve robust VAD. In a second stage, the TDOA estimates are directly used in the subband WRLS beamforming structure, without the need for covariance calculation. The subband beamformers are designed to

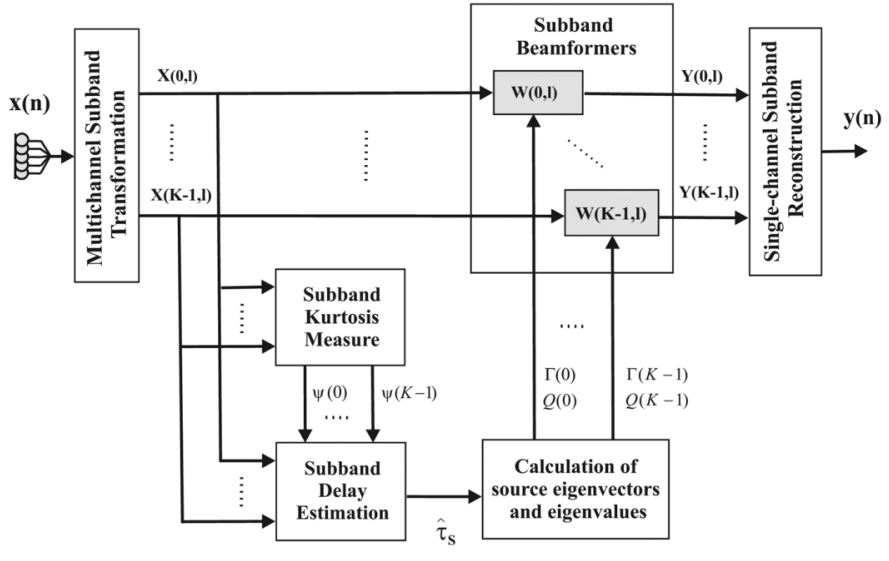

Fig. 2. Structure of proposed method.

provide frequency-dependent main lobe widths to match the frequency-dependent spatial resolution of the array. This structure takes into consideration the microphone's gain variations and phase discrepancies, and is fitted for any array geometry, as long as it fulfills a necessary condition on maximum intersensor distance. An efficient tracking of the desired speech source when the speaker is moving is further presented in this paper. The structure of the proposed speech enhancement method is shown in Fig. 2.

\section{A. Subband Kurtosis-Weighted Time-Delay Estimation}

The TDOA vector for the dominant speech source is extracted from the received noisy signals. The TDE is performed based on a weighted maximization of cross-correlations between received subband signals. A subband Kurtosis measure is used to quantify the speech content of the subband time slots, prior to the TDE, and weight accordingly the contribution of these subband time slots in the TDE. This algorithm consists in a multidimensional search function that has several local maxima in the case of overlapping speech signals. According to the assumption that the target source is dominant, the corresponding maxima will be singled out.

The following subband Kurtosis measure is used as a measure of non-Gaussianity:

$$
\Psi(k)=\frac{E\left\{\left|X_{c}(k, l)\right|^{4}\right\}}{E^{2}\left\{\left|X_{c}(k, l)\right|^{2}\right\}}-3 .
$$

Background noise most commonly displays a nearly Gaussian distribution with a Kurtosis value close to zero, while speech signals are super-Gaussian and display a positive Kurtosis value (an evaluation of the Kurtosis measure is presented later in this paper and depicted in Fig. 4).

A modified SRP-PHAT algorithm [1], [23] is used on the received subband short-time signals for a time slot of $L$ block sample vectors. The estimate of the TDOA vector $\hat{\boldsymbol{\tau}}_{s}=\left[0, \hat{\tau}_{2}, \hat{\tau}_{3}, \ldots, \hat{\tau}_{M}\right]^{T}$, is given by the weighted averaging of the subband TDOA vector estimates, following

$$
\arg \max _{\mathcal{T}}\left\{\sum_{k=0}^{K-1} \mathcal{P}\{\Psi(k)\} \sum_{p=1}^{M} \sum_{q=1}^{M} G_{p, q}(k) e^{j \Omega_{k}\left(\tau_{p}-\tau_{q}\right)}\right\}
$$


where

$$
\begin{aligned}
\boldsymbol{\tau} & =\left[0, \tau_{2}, \tau_{3}, \ldots, \tau_{M}\right]^{T} \\
\mathcal{P}\{x\} & = \begin{cases}x, & \text { if } x>1.5 \\
0, & \text { elsewhere }\end{cases}
\end{aligned}
$$

and

$$
G_{p, q}(k)=\frac{E\left\{X_{p}(k, l) X_{q}(k, l)^{*}\right\}}{\left|E\left\{X_{p}(k, l) X_{q}(k, l)^{*}\right\}\right|}
$$

is the normalized cross power spectrum of the sound signals received by the microphone pair $p$ and $q$ and for a time slot of $L$ block samples $\mathbf{X}(k, l)$. The averaging can additionally be performed over $B$ time blocks of $L$ samples to reduce the estimation errors.

\section{B. Delay-Based Constrained Beamformer}

Information about the target speech source location given by the TDOA vector estimate is used to calculate source covariance estimates, for each time slot of $L$ block samples, as

$$
\begin{aligned}
\tilde{\mathbf{R}}_{s}(k) & =P_{s}(k) \int_{\tau \in \Phi_{k}} e^{-j \Omega_{k}\left(\hat{\boldsymbol{\tau}}_{s}+\tau \mathbf{u}\right)} e^{-j \Omega_{k}\left(\hat{\boldsymbol{\tau}}_{s}+\tau \mathbf{u}\right)^{H}} d \tau \\
\tilde{\mathbf{r}}_{s}(k) & =P_{s}(k) \int_{\tau \in \Phi_{k}} e^{-j \Omega_{k}\left(\hat{\boldsymbol{\tau}}_{s}+\tau \mathbf{u}\right)} d \tau
\end{aligned}
$$

where $\mathbf{u}=[0,1,1, \ldots, 1]^{T}$. The dependency on the block index has been omitted for simplicity. The parameter $\Phi_{k}$ is the timedelay range in subband $k$, corresponding to a volume containing the source position, and is defined as

$$
\Phi_{k}=\left[-\frac{\phi(k)}{2}, \frac{\phi(k)}{2}\right] .
$$

A frequency-dependent delay uncertainty $\phi(k)=\mathcal{F}\left\{\Omega_{k}\right\}$ (where $\mathcal{F}$ is a monotonically increasing function) is chosen to adjust the beamformer's opening for each subband in order to reduce the source's speech distortion. The rationale behind this definition is that the beamformer (main lobe) spatial resolution increases with the subband frequency. It is also dependent on intermicrophone spacing as well as the source signal angle of arrival. The objective is to create a relatively homogeneous (i.e., frequency-independent) main lobe width. Furthermore, the low-frequency bands essentially contain noise power; thus, a small constraint area allows for more noise suppression in the low-frequency bands. On the other hand, a large enough constraint area in the upper bands secures the relatively undistorted passage of the desired speech signals.

The subband time-delay range $\Phi_{k}$ can be discretized into $N$ points, $\varphi_{k, n}, n=1,2, \ldots, N$, with equidistant interdelays given by $\Delta_{\tau}=(2 \phi(k) / N-1)$. By using the vector notation $\varphi_{k, n}=\varphi_{k, n} \mathbf{u}$, and replacing the integral in (27) by a sum, the source covariance matrix can be approximated by

$$
\begin{aligned}
\tilde{\mathbf{R}}_{s}^{\prime}(k) & =P_{\boldsymbol{s}}(k) \sum_{n=1}^{N} e^{-j \Omega_{k}\left(\hat{\boldsymbol{\tau}}_{s}+\boldsymbol{\varphi}_{k, n}\right)} e^{-j \Omega_{k}\left(\hat{\boldsymbol{\tau}}_{s}+\boldsymbol{\varphi}_{k, n}\right)^{H}} \Delta_{\tau} \\
& =P_{s}(k) \Delta_{\tau} \sum_{n=1}^{N} \mathbf{d}_{n}(k) \mathbf{d}_{n}(k)^{H}
\end{aligned}
$$

where the summation is performed over a vector multiplication of the attenuation-free steering vectors for a point source with TDOA delay vectors $\left(\hat{\tau}_{s}+\varphi_{k, n}\right), n=1,2 \ldots, N$, given by

$$
\begin{aligned}
\mathbf{d}_{n}(k) & =e^{-j \Omega_{k}\left(\hat{\boldsymbol{\tau}}_{s}+\boldsymbol{\varphi}_{k, n}\right)} \\
& =\operatorname{diag}\left(e^{-j \Omega_{k} \hat{\boldsymbol{\tau}}_{s}}\right) e^{-j \Omega_{k} \boldsymbol{\varphi}_{k, n} .}
\end{aligned}
$$

Let us denote the diagonal matrix function of the TDOA vector estimate by

$$
\mathbf{D}_{s}(k)=\operatorname{diag}\left(e^{-j \Omega_{k} \hat{\boldsymbol{\tau}}_{s}}\right)
$$

and the portion of the steering vector independent of the target source information as

$$
\mathbf{v}_{n}(k)=e^{-j \Omega_{k} \varphi_{k, n}} .
$$

We obtain the following formulation for the source covariance matrix estimate:

$$
\begin{aligned}
\tilde{\mathbf{R}}_{s}^{\prime}(k) & =P_{s}(k) \Delta_{\tau} \sum_{n=1}^{N} \mathbf{D}_{s}(k) \mathbf{v}_{n}(k) \mathbf{v}_{n}(k)^{H} \mathbf{D}_{s}(k)^{H} \\
& =P_{s}(k) \Delta_{\tau} \mathbf{D}_{s}(k)\left[\sum_{n=1}^{N} \mathbf{v}_{n}(k) \mathbf{v}_{n}(k)^{H}\right] \mathbf{D}_{s}(k)^{H} \\
& =P_{s}(k) \mathbf{D}_{s}(k) \mathbf{M}(k) \mathbf{D}_{s}(k)^{H}
\end{aligned}
$$

where the matrix

$$
\mathbf{M}(k)=\Delta_{\tau} \sum_{n=1}^{N} \mathbf{v}_{n}(k) \mathbf{v}_{n}(k)^{H}
$$

is the component in the source covariance matrix that is independent of the source information. This matrix corresponds to the source covariance matrix of a constraint area corresponding to a zero TDOA between the array elements for subband $k$. Let us represent the TDOA-null covariance matrix by its eigenvector matrix $\mathbf{Q}(k)$ and eigenvalue matrix $\Gamma(k)$

$$
\mathbf{M}(k)=\mathbf{Q}(k) \boldsymbol{\Gamma}(k) \mathbf{Q}(k)^{H} .
$$

Thus, the source covariance matrix eigen-decomposition $\left(\tilde{\mathbf{R}}_{s}^{\prime}(k)=\mathbf{Q}_{s}(k) \boldsymbol{\Gamma}_{s}(k) \mathbf{Q}_{s}(k)^{H}\right)$ elements are given by

$$
\begin{aligned}
\mathbf{Q}_{s}(k) & =\mathbf{D}_{s}(k) \mathbf{Q}(k) \\
\boldsymbol{\Gamma}_{\boldsymbol{s}}(k) & =P_{s}(k) \boldsymbol{\Gamma}(k) .
\end{aligned}
$$

Note that $\mathbf{Q}(k)$ and $\boldsymbol{\Gamma}(k)$ can be precalculated. In a similar manner, the cross covariance vector estimate is given by

$$
\tilde{\mathbf{r}}_{s}^{\prime}(k)=P_{s}(k) \Delta_{\tau} \sum_{n=1}^{N} \mathbf{D}_{s}(k) \mathbf{v}_{n}(k) .
$$

In this paper, we assume the subband source power spectrum $P_{s}(k)=1$, where 1 denotes the $M$ vector of ones. Hence, the speech spectrum is not colored by the beamformer's weights, while the subband weight vectors are designed to have constant amplitude in the look direction (i.e., flat spectrum). This assumption implies that the focus of the filtering operations is in the spatial domain, solely. One straight-forward extension 
could involve the compensation of speech spectrum by calculating $P_{s}(k)$. A subband Kurtosis approach for the SNR estimation of short-time speech signals was suggested in [29]. Another approach is the multichannel source spectral estimation method proposed in [30].

\section{Algorithm Summary}

\section{Initialization Phase:}

Prior to online processing, the following steps are run for each subband $k=0,1, \ldots, K-1$.

1) Initialize the inverse of the total covariance matrix $\hat{\mathbf{R}}(k, 0)^{-1}=\delta^{-1} \mathbf{I}_{M}$, where $\mathbf{I}_{M}$ is the unitary matrix of size $M \times M$, and $\delta$ is an initialization constant.

2) Define an $M \times M$ dummy variable matrix $\mathbf{D}$.

3) Choose a forgetting factor $0<\lambda<1$.

4) Define $N$ discrete time-delay points $\varphi_{k, n}$ spanning the frequency-dependent delay uncertainty range $\Phi_{k}$ (see (29)), as well as the related equidistant interdelay $\Delta_{\tau}$, and calculate the corresponding vectors $\varphi_{k, n}$ and $\mathbf{v}_{n}(k)$ from (33).

5) Calculate the covariance matrix $\mathbf{M}(k)$ following (35). The matrix $\mathbf{M}(k)$ is saved in memory in a diagonalized form, according to (36).

\section{Operation Phase:}

The sampled array input signals are decomposed each into a set of $K$ subband signals. The online processing of the algorithm is then stated as two parallel procedures:

(a) For every block of $L$ time samples and for each subband $k=0,1, \ldots, K-1$, (except for point 2 , which requires to combine all subband signals):

1) Calculate the Kurtosis value $\Psi(k)$ following (23) with

$$
E\left\{\left|X_{c}(k, l)\right|^{\alpha}\right\} \approx \frac{1}{L} \sum_{l=0}^{L-1}\left|X_{c}(k, l)\right|^{\alpha}, \quad \alpha=2,4 .
$$

2) Calculate the TDOA vector estimate $\hat{\boldsymbol{\tau}}_{s}$ according to (24)-(26).

3) Calculate the diagonal matrix $\mathbf{D}_{s}(k)$ based on the TDOA vector estimate $\hat{\boldsymbol{\tau}}_{s}$, following (32).

4) Calculate the estimated eigenvector matrix $\mathbf{Q}_{s}(k)$ and eigenvalue matrix $\boldsymbol{\Gamma}_{s}(k)$ of the source covariance matrix following (37) and (38). The eigenvectors and eigenvalues are denoted, respectively:

$$
\begin{aligned}
\mathbf{Q}_{s}(k) & =\left[\begin{array}{llll}
\mathbf{q}_{s, 1}(k), & \mathbf{q}_{s, 2}(k), & \ldots & \mathbf{q}_{s, M}(k)
\end{array}\right] . \\
\boldsymbol{\Gamma}_{s}(k) & =\operatorname{diag}\left(\left[\begin{array}{llll}
\gamma_{s, 1}(k), & \gamma_{s, 2}(k), & \ldots & \gamma_{s, M}(k)
\end{array}\right]\right) .
\end{aligned}
$$

5) Calculate the cross covariance vector estimate $\tilde{\mathbf{r}}_{s}^{\prime}(k)$ according to (39).

(b) The beamformer is run as an iterative procedure with the steps described below, for each subband $k=0,1, \ldots, K-1$, and for $l=1,2,3, \ldots$,

1) Update the inverse total covariance matrix $\hat{\mathbf{R}}(k, l)^{-1}$ based on the Woodbury's identity (see the Appendix) as shown by (41) at the bottom of the page, where $p=l(\bmod M)+1$ and the notation $\bmod$ represents the modulus function.

2) Calculate the weight vector $\mathbf{W}(k, l)$ as follows:

$$
\mathbf{W}(k, l)=\hat{\mathbf{R}}(k, l)^{-1} \tilde{\mathbf{r}}_{s}^{\prime}(k) .
$$

3) Calculate the output signal $Y(k, l)$ as follows:

$$
Y(k, l)=\mathbf{W}(k, l)^{H} \mathbf{X}(k, l) \text {. }
$$

The output from all subband beamformers are used in the reconstruction filter bank to create the time-domain output.

\section{Evaluation}

\section{A. Simulations}

Simulations are constructed so as to include the influence of background noise, interference, as well as room reverberation. The data was acquired using a linear array of four microphones uniformly spaced with 5 -cm spacing, and was gathered on a multichannel DAT recorder with a sampling rate of $12 \mathrm{kHz}$. The signal at each microphone was bandlimited to $5 \mathrm{kHz}$. The room used in the experiment is of size $(3 \mathrm{~m} \times 4 \mathrm{~m} \times 3 \mathrm{~m})$, with the microphone array placed in the center of the room. The room had a reverberation time $\mathrm{RT}_{60}$ (time required for reverberation energy to decay by $60 \mathrm{~dB}$ ) of $210 \mathrm{~ms}$. Fig. 3 illustrates the arrangement of the equipment for sound data acquisition. To simulate ambient noise, four loudspeakers were positioned at the corners of the room facing the wall and emitting independent noise signals simultaneously. The emitted noise was colored noise, prerecorded in a typical office room and corresponding to the noise generated by computer fans and air conditioners. The noise sound pressure level was approximately $63 \mathrm{~dB}[\mathrm{~A}]$ for scenarios with SNR $=0 \mathrm{~dB}$.

Two experimental setups were considered: The first setup consisted of a target source, simulated by a loudspeaker at a fixed position, e.g., position (1) in Fig. 3 at $\left(50 \mathrm{~cm}, 0^{\circ}\right)$, and an interference source positioned at $\left(1 \mathrm{~m},-45^{\circ}\right)$. The sound used for the target source was female speech, while the interfering loudspeaker emitted male speech. The second setup was defined for a target source moving from position (1) described above along a quarter-circle path centered at the reference point. The

$$
\begin{aligned}
\mathbf{D} & =\lambda^{-1} \hat{\mathbf{R}}(k, l-1)^{-1}-\frac{\lambda^{-2} \hat{\mathbf{R}}(k, l-1)^{-1} \mathbf{X}(k, l) \mathbf{X}(k, l)^{H} \hat{\mathbf{R}}(k, l-1)^{-1}}{1+\lambda^{-1} \mathbf{X}(k, l)^{H} \hat{\mathbf{R}}(k, l-1)^{-1} \mathbf{X}(k, l)} \\
\hat{\mathbf{R}}(k, l)^{-1} & =\mathbf{D}-\frac{(1-\lambda) \gamma_{s, p}(k) \mathbf{D} \mathbf{q}_{s, p}(k) \mathbf{q}_{s, p}(k)^{H} \mathbf{D}}{1+(1-\lambda) \gamma_{s, p}(k) \mathbf{q}_{s, p}(k)^{H} \mathbf{D} \mathbf{q}_{s, p}(k)}
\end{aligned}
$$




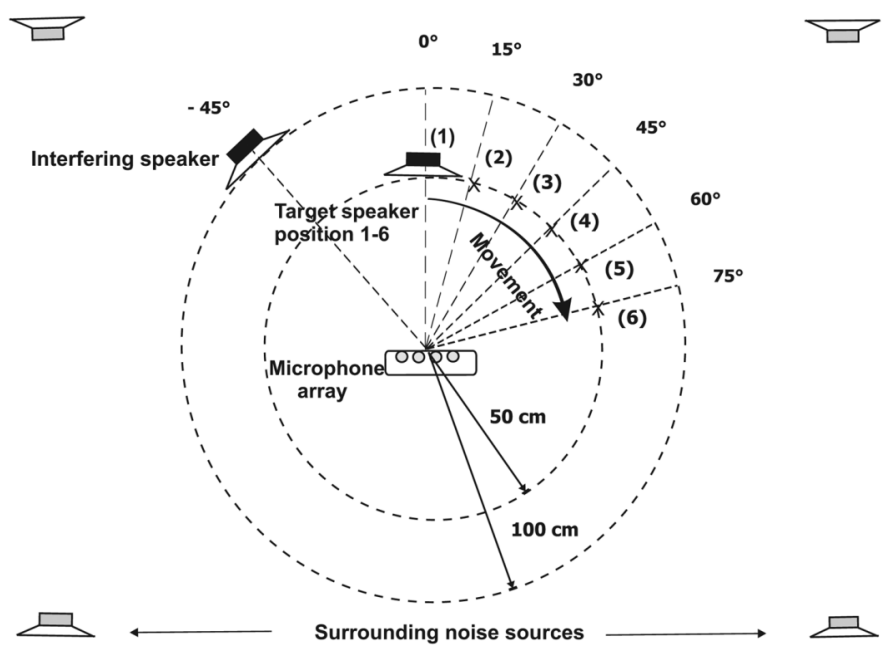

Fig. 3. Configuration of microphone array and sound sources in an office room. The source movement path is represented by the numbered crosses, which are passed at a speed of one second per step (i.e., $15^{\circ} / \mathrm{s}$ ).

update of the SRP-PHAT algorithm took place at the speaker positions represented by the numbered crosses in Fig. 3. The time taken to move between two adjacent speaker positions was one second (i.e., a movement of $15^{\circ} / \mathrm{s}$ ).

\section{B. Subband Decomposition}

The subband decomposition of the microphone input signals is achieved by means of a multichannel modulated uniform analysis filter bank [26]. The spatial characteristics of the input signal are maintained by using the same filter bank at each microphone. The subband outputs of the subsequent spatial filters are reconstructed by a modulated uniform synthesis filter bank, in order to create a time-domain output signal. A resulting computational gain of subband processing arises due to the fact that the processing of narrow-band signals requires lower sample rates. Hence, in an efficient implementation, the frequency transform is followed by a decimation operation [26]. A simplified structure is possible through the use of an efficient polyphase implementation. For a detailed discussion of the properties of this transform, see [5].

\section{Evaluation of the Subband Kurtosis Measure}

This section evaluates the subband Kurtosis measure of (23) and (40) for different types of sound sources (speech, music, recorded fan noise, and Gaussian-distributed white noise). The sound sequences consist of recordings of speech sequences emitted by the target loudspeaker at position (1) in the absence of noise and interference, and gathered by each sensor. The speech sequences were selected from two female and two male continuous speech segments taken from the TIMIT database. Additionally, sequences of music, recorded fan noise, and artificial Gaussian-distributed white noise were individually emitted by the surrounding loudspeakers and recorded separately. The relation between the number of subbands $K$ and the mean subband Kurtosis measure for the different signals is given in Fig. 4. The length of the sequences over which the measure is performed is set to $T_{k}=0.5 \mathrm{~s}$ and the plots are performed for an average of 100 sound sequences. The standard

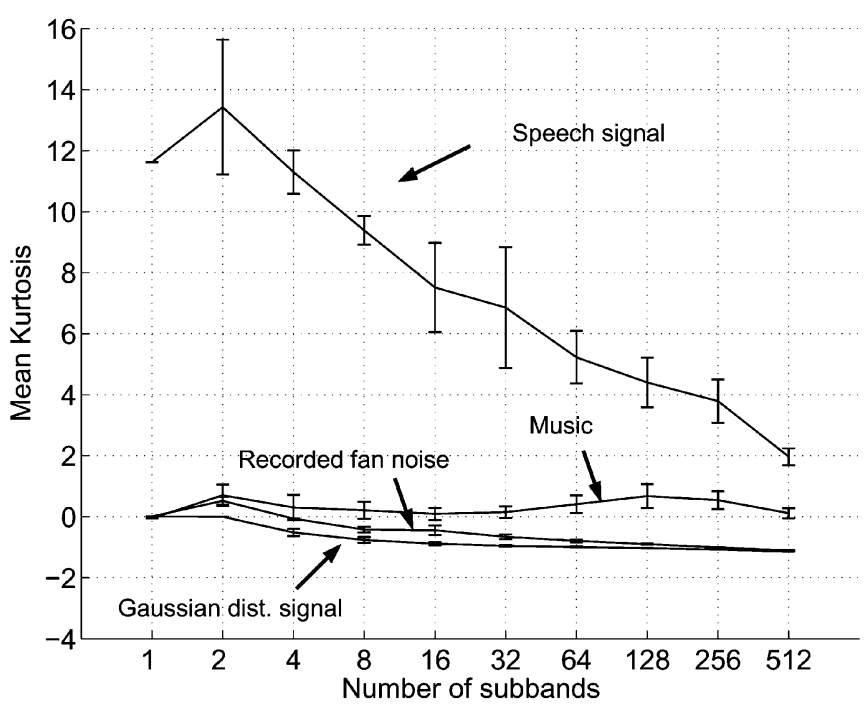

Fig. 4. Evaluation of the subband Kurtosis measure presented in Section IV-A versus the number of subbands, for different sound types. The sound sequence length is fixed to $T_{k}=0.5 \mathrm{~s}$. The vertical lines correspond to the standard deviation of the measurements at each point.

deviation of the data used for each average is also displayed as bars centered over the mean value. It can be seen that speech presents a positive mean Kurtosis value over all subbands, while noise signals exhibit a predominantly negative Kurtosis. Music sequences are attested with positive, close to zero values. The observations above yield a method of distinguishing subband sound sequences with speech content from noise and music sequences.

In this experiment, the decimation factor used in the subband decomposition of the received signal was set to $K / 2$. Hence, it follows that the number of data $L$ over which the averaging of (40) is done is inversely proportional to $K$ (i.e., $L=2 T_{k} F_{s} / K$ ). This justifies the decrease in the Kurtosis measure towards the value of -2 when increasing $K$ for all signal types (i.e., when $L$ goes towards $1, \Psi(k) \approx-2$ ). The following simulations are performed with 64 subbands.

\section{Evaluation of Proposed Subband Kurtosis-Weighted TDE Method}

In this section, we focus on demonstrating that the proposed subband Kurtosis-weighted TDE method presented in Section IV-A is more robust and performs better than the standard SRP-PHAT method [1], [23]. In Fig. 5, the TDE of these two methods is plotted for different DOA angles relative to the linear array. A speech sequence of length $2 \mathrm{~s}$ was generated sequentially from the six positions (1 to 6) of Fig. 3, while colored noise was emitted from the four surrounding loudspeakers. The noisy speech sequence was recorded with SNR $=-10 \mathrm{~dB}$. In plot (c), the TDE was performed every $T_{k}=0.5 \mathrm{~s}$, and in plot (d), $T_{k}=0.25 \mathrm{~s}$. The obtained results show a much more robust DOA estimation using the proposed method. The DOA estimation points were computed using short-time segments in which speech power was low compared to noise power, explaining the erroneous estimates obtained using the standard SRP-PHAT method. The robustness of the subband-weighted approach derives from the fact that the subbands of the sound 

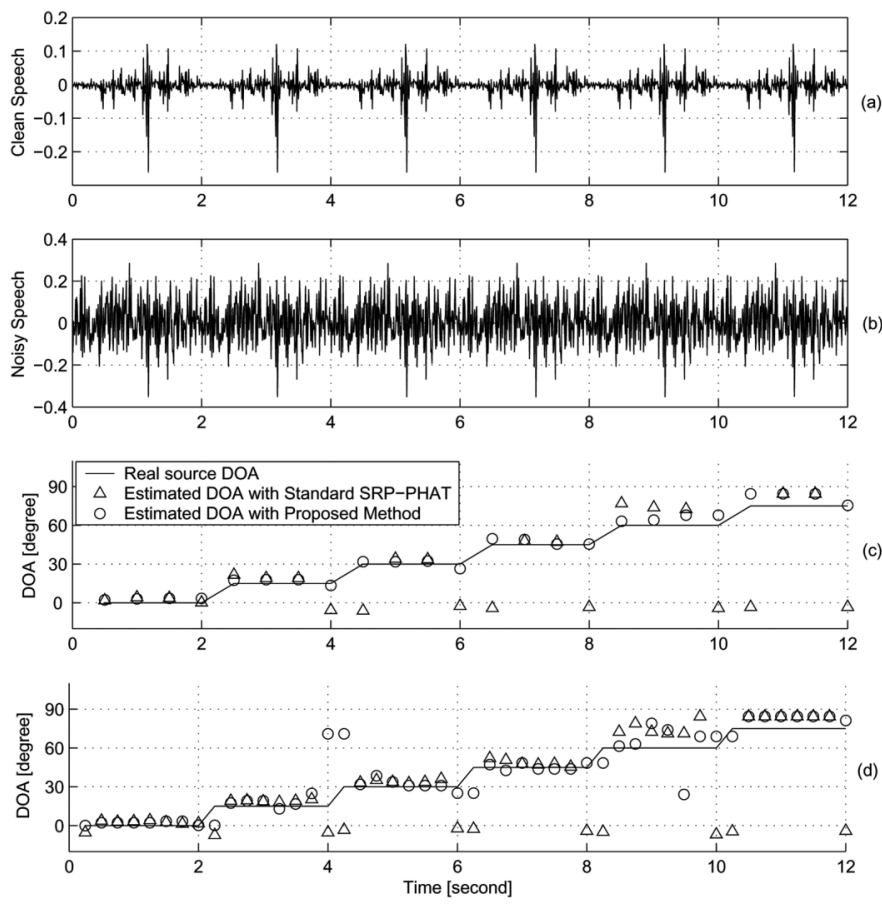

Fig. 5. Comparison of the proposed subband Kurtosis-weighted TDE method to the standard SRP-PHAT method for an instantaneous speech sequence and versus different DOA angles (bottom two graphs). The graphs correspond to (a) clean speech sequence received at the microphone array, (b) noisy sequence (SNR $=-10 \mathrm{~dB})$ recorded by the microphone array, (c) DOA estimates calculated by the standard SRP-PHAT and the proposed TDE method every $T_{k}=$ $0.5 \mathrm{~s}$, and (d) DOA estimates for $T_{k}=0.25 \mathrm{~s}$.

segments with higher speech content contribute more to the estimation process. On the other hand, the subbands with a low, or no, speech content (i.e., high noise power content) will be discarded. The results with smaller time-frame length, $T_{k}=0.25 \mathrm{~s}$, show higher variance in the TDOA estimation of the proposed method, as expected, but at acceptable levels for the following constrained beamforming.

The two methods mentioned above are further compared in Fig. 6, for different short-time sequence lengths of the sound signals used in the DOA estimation $\left(T_{k}=0.25 \mathrm{~s}, 0.5 \mathrm{~s}\right.$, and $1 \mathrm{~s})$. The error in source DOA estimation, averaged over 600 noisy speech sequences acquired by the microphone array ( 25 segments $\times$ four speakers $\times$ six DOA positions), is plotted for different SNR values. It can be seen that the subband Kurtosisweighted approach to TDE provides up to $10^{\circ}$ better DOA estimation at low SNR and performs better than the standard SRPPHAT approach even at high SNR.

\section{E. Evaluation of Proposed Subband Beamformer}

In order to evaluate the proposed beamformer, the weights were calculated for the first setup according to (42), with $\hat{\mathbf{R}}(k, l)^{-1}$ recursively updated following (41). The constraint characteristics were precalculated following (33), (35), and (36). The proposed subband TDE method described in Section IV-A was used to estimate the TDE vector $\boldsymbol{\tau}_{s}$. The source covariance information was then estimated according to (32), (37), (38), and (39). The forgetting factor was chosen as $\lambda=0.99$, and the initialization constant as $\delta=0.01$.

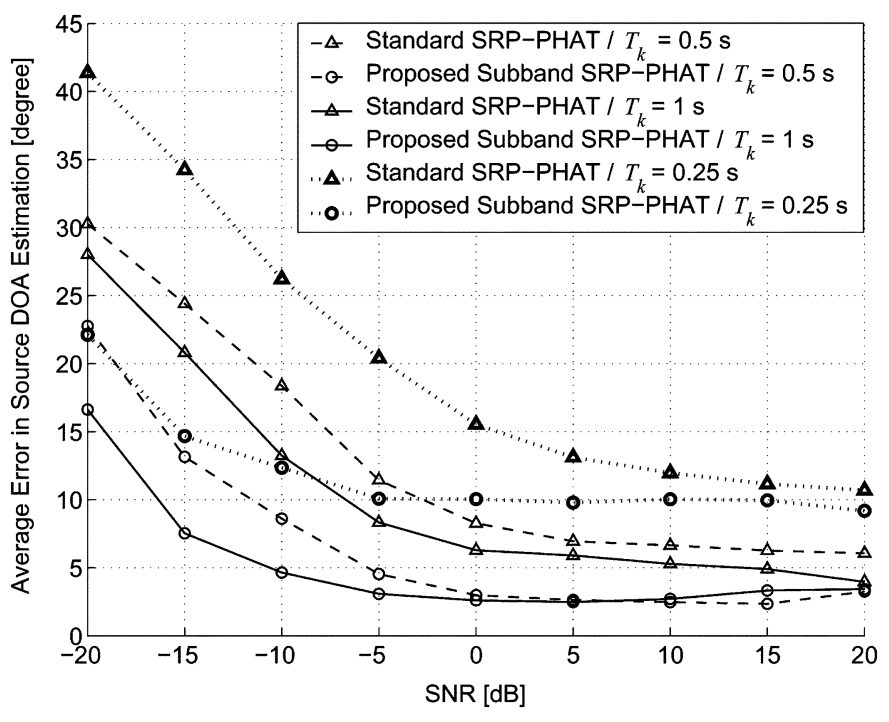

Fig. 6. Evaluation of the subband Kurtosis-weighted delay estimation method for different SNR values.

TABLE I

PERFormance MEASURES FOR DifFERENT VALUES OF THE NuMbER OF SubBands $K$ AND FOR THE TARget SPEAKER AT Position (3). $\mathrm{SNR}=-5 \mathrm{~dB}$ AND SIR $=3 \mathrm{~dB}$

\begin{tabular}{|c|c|c|c|}
\hline No. subbands & Distortion [dB] & Noise sup. [dB] & Interf. sup. [dB] \\
\hline 8 & -33.1 & 11.8 & 5.5 \\
\hline 16 & -33.2 & 13.2 & 5.8 \\
\hline 32 & -33.1 & 16.7 & 10.5 \\
\hline 64 & -34.4 & 16.0 & 16.1 \\
\hline 128 & -34.8 & 14.8 & 16.6 \\
\hline 256 & -35.12 & 13.2 & 14.3 \\
\hline 512 & -36.4 & 9.7 & 8.6 \\
\hline
\end{tabular}

The performance evaluation was based on three measures, the noise and interference suppression and the speech source distortion caused by the beamforming filters. The normalized distortion quantity $\mathcal{D}$, measured by the spectral deviation between the beamformer output and the source signal, is introduced as

$$
\mathcal{D}=\frac{1}{2 \pi} \int_{-\pi}^{\pi}\left|C_{d} \hat{P}_{y_{s}}(\Omega)-\hat{P}_{x_{s}}(\Omega)\right| d \Omega .
$$

The constant $C_{d}$ is defined as

$$
C_{d}=\frac{\int_{-\pi}^{\pi} \hat{P}_{x_{s}}(\Omega) d \Omega}{\int_{-\pi}^{\pi} \hat{P}_{y_{s}}(\Omega) d \Omega}
$$

where $\hat{P}_{x_{s}}(\Omega)$ is the PSD estimate of a single sensor observation, and $\hat{P}_{y_{s}}(\Omega)$ is the PSD estimate of the beamformer output, when the source signal is active alone. The constant $C_{d}$ normalizes the mean output PSD estimate to that of the single-sensor mean PSD estimate. The single-sensor observation is chosen as the reference microphone observation. The measure of distortion in (44) is the mean output PSD deviation from the observed single-sensor power spectral density estimate. Ideally, the distortion is zero $(-\infty \mathrm{dB})$.

1) Performance Versus Spectral Resolution: In Table I, the speech distortion, as well as the noise and interference suppression measures, are shown as a function of the number of subbands $K$. Results show that highest levels of noise plus inter- 

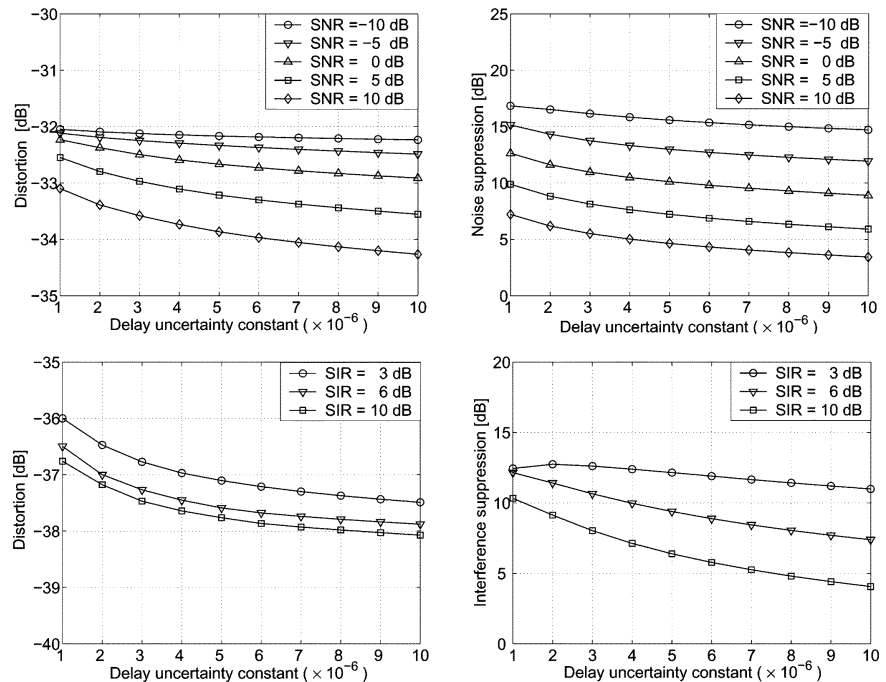

Fig. 7. Performance measures of the proposed beamformer versus the delay uncertainty constant $\mathcal{T}$, for different SNR values (top plots) with the interference loudspeaker silent, and for different SIR values (bottom plots) with no background noise present. The target speaker is at position (3).

ference suppression of the proposed method are obtained when using about 64 subbands. The reason is that a tradeoff between the beamformer's performance and the Kurtosis-weighted TDE accuracy is obtained by varying the number of subbands. This is due to the fact that the Kurtosis measure needs more data (i.e., higher $L$ ) to provide results with a lower variance. Hence, since $L$ is inversely proportional to $K$ for a fixed sequence length, the variance of the Kurtosis increases with $K$.

2) Performance Versus Constraint Region Size: The source covariance matrix was obtained for a constraint region defined by a delay uncertainty $\phi_{k}=\mathcal{T} \Omega_{k}^{1 / 4}$ and centered at the position of the source given by the tracking algorithm, where $\mathcal{T}$ is a delay-uncertainty constant. The number of chosen discrete points in the subband time-delay range $\Phi_{k}$ is $N=10$.

Fig. 7 shows the algorithm performance for different constraint region sizes by varying the delay uncertainty constant $\mathcal{T}$. Results are plotted for different SNR and signal-to-interference (SIR) values. For $\mathcal{T}=10^{-6}$ in the horizontal axes, the DOA-spread range is $\left[1.6^{\circ}, 5.5^{\circ}\right]$ (i.e., the smallest constraint corresponds to a DOA-spread of $1.6^{\circ}$ for the lowest frequency band and $5.5^{\circ}$ for the highest band), while for $\mathcal{T}=10^{-5}$ (largest constraint), the DOA-spread range is $\left[16.5^{\circ}, 73.7^{\circ}\right]$. It can be seen that a tradeoff between noise (and interference) suppression and speech distortion is made possible through the choice of the constraint delay-spread. A smaller delay-spread results in more noise and interference suppression at the expense of some additional speech distortion. A major benefit of this approach is the adaptation of the noise and interference suppression levels to the SNR and SIR values. In fact, the more noise or interference is present, the higher suppression is accomplished with a relatively small increase in speech distortion.

3) Performance Versus Source Movement: The impact of the source movement on the beamformer performance was evaluated using the second setup. Fig. 8 shows the noise and interference suppression and speech distortion measures for the different positions given by the tracking algorithm and corresponding to the speaker's movement. The performance measures are plotted for small and large constraint areas (top plots) and for different SNR values (bottom plots). A delay-uncertainty constant $\mathcal{T}=3 \times 10^{-6}$ was chosen for the second set of simulations. It corresponds to a frequency-dependant DOA-spread of $5^{\circ}$ for the lowest frequency band and $16.6^{\circ}$ for the highest band. The proposed solution exhibits a good tracking capability when the speaker is moving. As the angle of the source increases, corresponding to a movement away from the front view of the array, more noise is suppressed with an increase in speech distortion. Additionally, the further apart the source and the interference loudspeakers are, the more interference suppression is achieved. A larger constraint area provides less distortion at the expense of smaller noise and interference suppression.

4) Comparative Performance: A comparison of the distortion and suppression levels obtained for different optimal and adaptive beamformers listed in Table II is presented in Fig. 9 for different SNR conditions. Also, the waveforms of the proposed and conventional beamformers (at SNR $=-5 \mathrm{~dB}$ ) are plotted for comparison in Fig. 10.

The optimal weights of the maximum signal-to-noise-plusinterference ratio beamformer (SNIB) are calculated according to

$$
\mathbf{W}_{\mathrm{SNIB}}(k)=\arg \max _{\mathbf{W}}\left(\frac{\mathbf{W}^{H} \hat{\mathbf{R}}_{s}(k) \mathbf{W}}{\mathbf{W}^{H}\left(\hat{\mathbf{R}}_{i}(k)+\hat{\mathbf{R}}_{n}(k)\right) \mathbf{W}}\right)
$$

where

$$
\begin{aligned}
\hat{\mathbf{R}}_{s}(k) & =\frac{1}{L} \sum_{l=0}^{L-1} \mathbf{X}_{s}(k, l) \mathbf{X}_{s}(k, l)^{H} \\
\hat{\mathbf{R}}_{i}(k) & =\frac{1}{L} \sum_{l=0}^{L-1} \mathbf{X}_{i}(k, l) \mathbf{X}_{i}(k, l)^{H} \\
\hat{\mathbf{R}}_{n}(k) & =\frac{1}{L} \sum_{l=0}^{L-1} \mathbf{X}_{n}(k, l) \mathbf{X}_{n}(k, l)^{H}
\end{aligned}
$$

are the source, interference, and noise deterministic covariance matrices, respectively.

The LS beamformer weights are calculated according to (12), where the following deterministic spatial covariances are used:

$$
\begin{aligned}
& \hat{\mathbf{R}}_{x}(k)=\frac{1}{L} \sum_{l=0}^{L-1} \mathbf{X}(k, l) \mathbf{X}(k, l)^{H} \\
& \hat{\mathbf{r}}_{\mathbf{x} s}(k)=\frac{1}{L} \sum_{l=0}^{L-1} \mathbf{X}(k, l) S(k, l)^{*} .
\end{aligned}
$$

The adaptive beamformers described in Sections III and IV are evaluated by storing the source information in terms of the diagonalized covariance matrices, and by continuously adapting the weights and creating the output for the real speech recordings following (41)-(43). For the calibrated beamformer, CC-WRLS, the source information is calculated from noise-free source calibration sequences, according to (17) and (18). For the model-based and delay-based beamformers, it is estimated for propagation models of the source, as defined by [(21), (22)] 

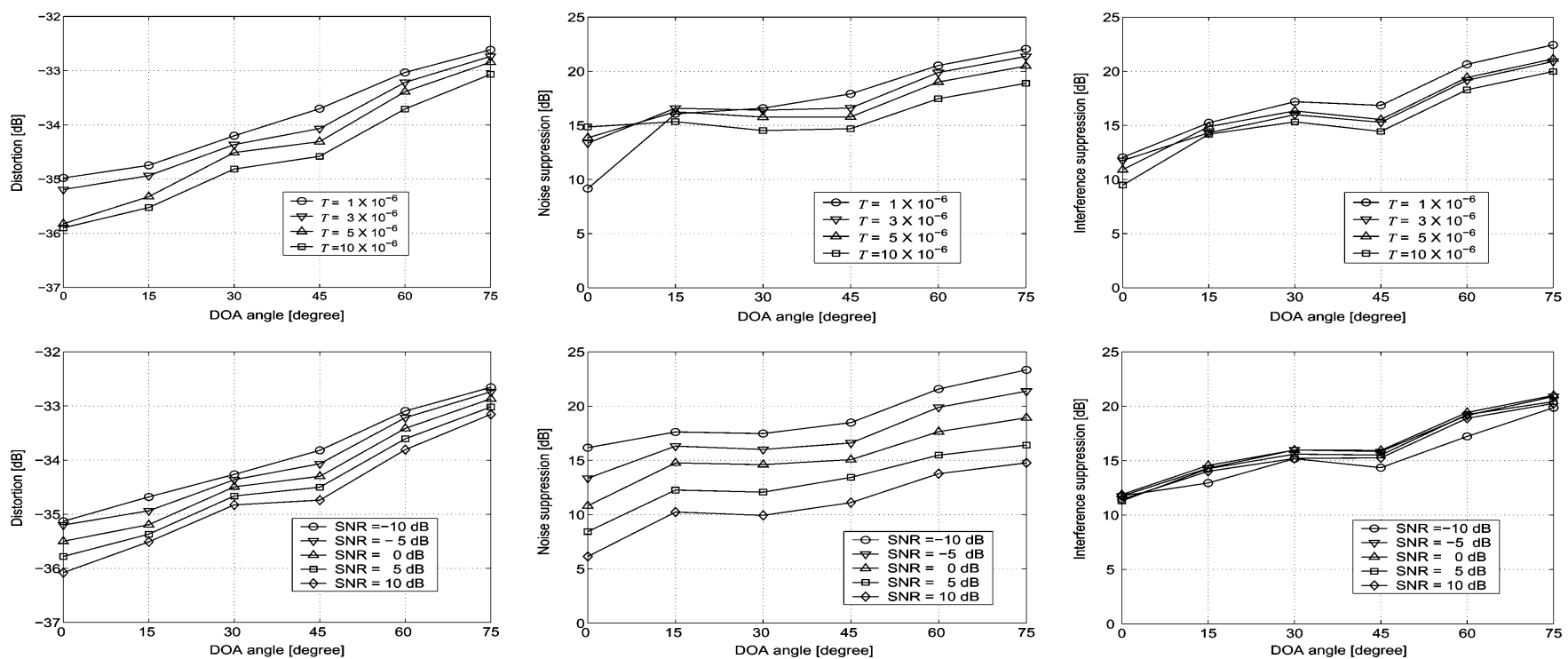

Fig. 8. Performance measures of the proposed beamformer for a moving target. The results are plotted versus the DOA angle of the desired source signal to the linear array, which corresponds to the source's movement of $15^{\circ} / \mathrm{s}$. The upper plots are given for different values of the delay uncertainty constant $\mathcal{T}$, with $\mathrm{SNR}=-5 \mathrm{~dB}$ and $\mathrm{SIR}=3 \mathrm{~dB}$. The lower plots are given for different SNR values, with $\mathrm{SIR}=3 \mathrm{~dB}$ and $\mathcal{T}=3 \times 10^{-6}$.
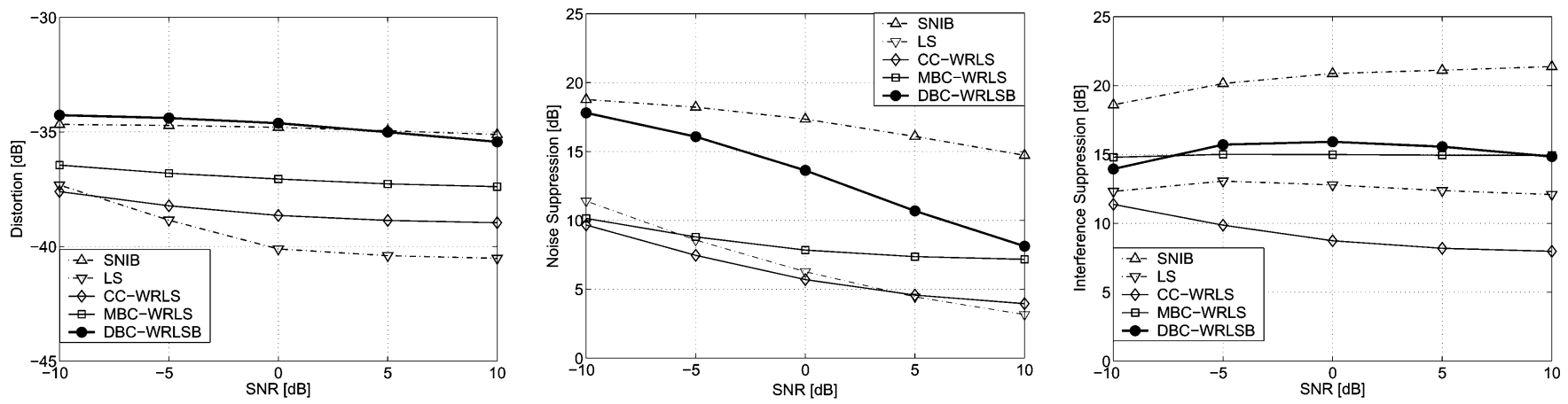

Fig. 9. Performance measures for the optimal and adaptive subband beamforming algorithms listed in Table II, with a nonmoving target speaker at position (3) and $\mathrm{SIR}=3 \mathrm{~dB}$.

TABLE II

List OF THE COMPARED OPTIMAL AND ADAPTIVE SUBBAND BEAMFORMING ALGORITHMS

\begin{tabular}{|c|c|c|}
\hline $\begin{array}{c}\text { Algorithm } \\
\text { Category }\end{array}$ & $\begin{array}{c}\text { Subband } \\
\text { Beamformer }\end{array}$ & $\begin{array}{l}\text { Algorithm } \\
\text { Equations }\end{array}$ \\
\hline \multirow{2}{*}{$\begin{array}{c}\text { Optimal } \\
\text { Non-Blind }\end{array}$} & SNIB & $(46)-(47),(48),(49)$ \\
\hline & LS & $(12)-(50),(51)$ \\
\hline \multirow{2}{*}{$\begin{array}{c}\text { Adaptive } \\
\text { Non-Blind }\end{array}$} & CC-WRLS & $(41),(16)-(17),(18)$ \\
\hline & MBC-WRLS & $(41),(16)-(21),(22)$ \\
\hline $\begin{array}{l}\text { Adaptive } \\
\text { Blind }\end{array}$ & $\begin{array}{c}\text { Proposed } \\
\text { DBC-WRLSB }\end{array}$ & $\begin{array}{l}(41),(42)-(32),(37),(38),(39) \\
\text { with precalculated }(33),(35),(36)\end{array}$ \\
\hline
\end{tabular}

and [(32), (37)-(39)], respectively. Both methods rely on the proposed subband weighted TDE method of Section IV-A for the estimation of the TDE vector $\tau_{s}$.

In comparison to the optimal LS beamformer, it can be seen that the optimal SNIB beamformer has better suppression levels of both noise and interference. However, the LS beamformer distorts the input source signal to a lesser degree. It can also be seen that the model-based constrained beamformer, MBC-WRLS, presents better suppression levels then the calibration-based constrained beamformer, CC-WRLS, with additional distortion. The proposed delay-based constrained beamformer, DBC-WRLSB, provides performance closer to the optimal SNIB beamformer in comparison to the previously suggested adaptive beamformers, in addition to being blind.

\section{CONCLUSION}

A new blind subband beamforming approach for moving source speech enhancement has been presented, together with an evaluation conducted in an office room. The proposed beamformer is a recursive least squares algorithm, using a soft-constraint defined for a delay-spread corresponding to a volume around the speech source location. A new speech-oriented TDE algorithm is combined with the beamformer to allow for speaker movement. The update of the soft-constraint due to source mobility is performed recursively. The proposed method does not require any calibration data, knowledge of the array manifold, or any other acoustical environment characteristics. Hence, it provides means to blindly enhance a dominant speaker in adverse noise conditions. The real room evaluation shows up to $16-\mathrm{dB}$ noise reduction and 16- $\mathrm{dB}$ interference suppression with a perceptually low speech distortion. 

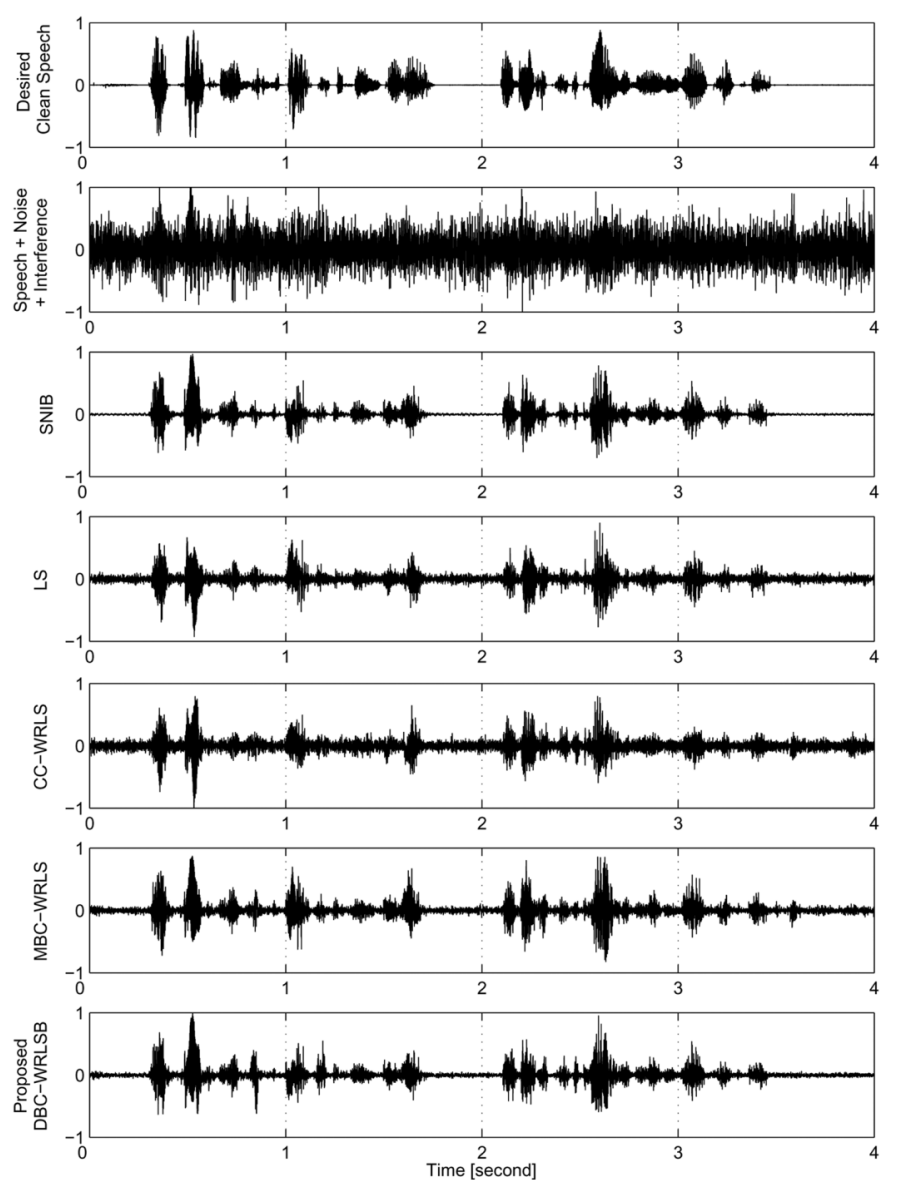

Fig. 10. Comparison of normalized input and output signals to the proposed and conventional beamformers. The target speaker is at position (3). SNR = $-5 \mathrm{~dB}$ and $\mathrm{SIR}=3 \mathrm{~dB}$

\section{APPENDIX}

Given that the time-dependent parameter on the right-hand side of (16) can be expressed recursively (from (15)) based on the available input data vector $\mathbf{X}(k, l)$ as

$$
\hat{\mathbf{R}}_{\mathbf{x}}(k, l)=\lambda \hat{\mathbf{R}}_{\mathbf{x}}(k, l-1)+\mathbf{X}(k, l) \mathbf{X}(k, l)^{H}
$$

a recursive solution for the update of the beamforming weight vector $\mathbf{W}(k, l)$ is derived. Since we are interested in the inverse of

$$
\begin{aligned}
\hat{\mathbf{R}}(k, l)= & \mathbf{R}_{s}(k)+\hat{\mathbf{R}}_{\mathbf{x}}(k, l) \\
= & \mathbf{R}_{s}(k)+\lambda \hat{\mathbf{R}}_{\mathbf{x}}(k, l-1)+\mathbf{X}(k, l) \mathbf{X}(k, l)^{H} \\
= & \mathbf{R}_{s}(k)-\lambda \mathbf{R}_{s}(k)+\lambda\left[\mathbf{R}_{s}(k)+\hat{\mathbf{R}}_{\mathbf{x}}(k, l-1)\right] \\
& +\mathbf{X}(k, l) \mathbf{X}(k, l)^{H} \\
= & (1-\lambda) \mathbf{R}_{s}(k)+\lambda \hat{\mathbf{R}}(k, l-1)+\mathbf{X}(k, l) \mathbf{X}(k, l)^{H}
\end{aligned}
$$

the inversion of the total sum is required, including the precalculated correlation matrix $\mathbf{R}_{s}(k)$. An alternative approach, in order to reduce the complexity of the problem, is to simplify the representation of these matrices in (53), by applying the spectral theorem, to the form

$$
\begin{aligned}
\mathbf{R}_{s}(k) & =\mathbf{Q}_{s}(k) \boldsymbol{\Gamma}_{s}(k) \mathbf{Q}_{s}(k)^{H} \\
& =\sum_{p=1}^{M} \boldsymbol{\gamma}_{s, p}(k) \mathbf{q}_{s, p}(k) \mathbf{q}_{s, p}(k)^{H} .
\end{aligned}
$$

Here, $\mathbf{Q}_{s}(k)=\left[\mathbf{q}_{s, 1}(k), \mathbf{q}_{s, 2}(k), \ldots, \mathbf{q}_{s, M}(k)\right]$ is the eigenvector matrix and $\boldsymbol{\Gamma}_{s}(k)=\operatorname{diag}\left(\left[\boldsymbol{\gamma}_{s, 1}(k), \boldsymbol{\gamma}_{s, 2}(k), \ldots\right.\right.$, $\left.\left.\gamma_{s, M}(k)\right]\right)$ the eigenvalue matrix of $\mathbf{R}_{s}(k)$. This will result in adding a sum of scaled eigenvectors of the source correlation matrix to the update of (53), corresponding to several rank-one updates. Also, by sequentially adding one scaled eigenvector for each sample $l$, it allows for the use of the Woodbury's identity in the inversion of $\hat{\mathbf{R}}(k, l)$. By doing so, the complexity is further reduced while only affecting the scale of the problem [21]. We obtain the following expression for the update of $\hat{\mathbf{R}}(k, l)$ :

$$
\begin{aligned}
\hat{\mathbf{R}}(k, l)= & \lambda \hat{\mathbf{R}}(k, l-1)+\mathbf{X}(k, l) \mathbf{X}(k, l)^{H} \\
& +(1-\lambda)\left[\boldsymbol{\gamma}_{s, p}(k) \mathbf{q}_{s, p}(k) \mathbf{q}_{s, p}(k)^{H}\right]_{p=l(\bmod M)+1}
\end{aligned}
$$

\section{REFERENCES}

[1] M. Brandstein and D. Ward, Microphone Arrays—Signal Processing Techniques and Applications. New York: Springer, 2001.

[2] D. Johnson and D. Dudgeon, Array Signal Processing-Concepts and Techniques. Englewood Cliffs, NJ: Prentice-Hall, 1993.

[3] A. Bell and T. Sejnowski, "An information maximization approach to blind separation and blind deconvolution," in Neural Comput., Nov. 1995, vol. 7, pp. 1129-1159.

[4] P. Smaragdis, "Efficient blind separation of convolved sound mixtures," in Proc. IEEE Applicat. Signal Process. Audio Acoust., 1997, pp. 19-22.

[5] N. Grbić, X. J. Tao, S. Nordholm, and I. Claesson, "Blind signal separation using overcomplete subband representation," IEEE Trans. Speech Audio Process., vol. 9, no. 5, pp. 524-533, Jul. 2001.

[6] S. Araki, R. Mukai, S. Makino, T. Nishikawa, and H. Saruwatari, "The fundamental limitation of frequency domain blind source separation for convolutive mixtures of speech," IEEE Trans. Speech Audio Process. vol. 11, no. 2, pp. 109-116, Mar. 2003.

[7] S. Y. Low, S. Nordholm, and R. Togneri, "Convolutive bind signal separation with post-processing," IEEE Trans. Speech Audio Process., vol. 12, no. 5, pp. 439-548, Sep. 2004.

[8] R. Mukai, S. Araki, H. Sawada, and S. Makino, "Removal of residual cross-talk components in blind source separation using time-delayed spectral substraction," in Proc. IEEE Int. Conf. Acoust., Speech, Signal Process., 2002, vol. 2, pp. 1789-1792.

[9] H. Sawada, S. Araki, R. Mukai, and S. Makino, "Blind extraction of a dominant source signal from mixtures of many sources," in IEEE Proc. Int. Conf. Acoust., Speech, Signal Process., May 2005, vol. 3, pp. 61-64.

[10] H. Saruwatari, T. Kawamura, T. Nishikawa, A. Lee, and K. Shikano, "Blind signal separation based on a fast-convergence algorithm combining ICA and beamforming," IEEE Trans. Audio, Speech, Lang. Process., vol. 14, no. 2, pp. 666-678, Mar. 2006.

[11] J.-F. Cardoso, "Blind source separation: Statistical principles," Proc. IEEE, vol. 86, no. 10, pp. 2009-2025, Oct. 1998.

[12] A. Hyväringen, J. Karhunen, and E. Oja, Independent Component Analysis. New York: Wiley, 2001.

[13] J. P. LeBlanc and P. L. De Léon, "Speech separation by Kurtosis maximization," in IEEE Proc. Int. Conf. Acoust., Speech, Signal Process., May 1998, vol. 2, pp. 1029-1032.

[14] B. W. Gillespie, H. S. Malvar, and D. A. F. Florêncio, "Speech dereverberation via maximum-Kurtosis subband adaptive filtering," in IEEE Proc. Int. Conf. Acoust., Speech, Signal Process., May 2001, vol. 6, pp. 3701-3704 
[15] Ö. Yilmaz and S. Rickard, "Blind separation of speech mixtures via time-frequency masking," IEEE Trans. Signal Process., vol. 52, no. 7, pp. 1830-1847, Jul. 2004.

[16] N. Grbić, S. Nordholm, and A. Cantoni, "Optimal FIR subband beamforming for speech enhancement in multipath environments," IEEE Signal Process. Lett., vol. 10, no. 11, pp. 335-338, Nov. 2003.

[17] S. Shahbazpanahi, A. B. Gershman, Z. Q. Luo, and K. M. Wong, "Robust adaptive beamforming for general-rank signal model," IEEE Trans. Signal Process., vol. 51, no. 9, pp. 2257-2269, Sep. 2003.

[18] S. Affes and Y. Grenier, "A signal subspace tracking algorithm for microphone array processing of speech," in Proc. Int. Conf. Acoust., Speech, Signal Process., Sep. 1997, vol. 5, pp. 425-437.

[19] H. Gustafsson, S. Nordholm, and I. Claesson, "Spectral substraction using reduced delay convolution in adaptive averaging," IEEE Trans. Speech Audio Process., vol. 9, no. 8, pp. 799-807, Nov. 2001.

[20] S. Nordholm, I. Claesson, and M. Dahl, "Adaptive microphone array employing calibration signals: An analytical evaluation," IEEE Trans. Speech Audio Process., vol. 7, no. 3, pp. 241-252, May 1999.

[21] Z. Yermeche, P. M. Garcia, N. Grbić, and I. Claesson, "A calibrated subband beamforming algorithm for speech enhancement," in Proc. IEEE Sensor Array Multichannel Signal Process. Workshop, Aug. 2002, pp. 485-489.

[22] Z. Yermeche, N. Grbić, and I. Claesson, "Beamforming for moving source speech enhancement," in Proc. IEEE Worshop Applicat. Signal Process. Audio Acoust., Oct. 2005, pp. 25-28.

[23] Z. Yermeche, N. Grbić, and I. Claesson, "Moving source speech enhancement using time-delay estimation," in Proc. Int. Worshop Acoust. Echo Noise Control, Sep. 2005, pp. 69-72.

[24] E. Nemer, R. Goubran, and S. Mahmoud, "Robust voice activity detection using higher-order statistics in the LPC residual domain," IEEE Trans. Speech Audio Process., vol. 9, no. 3, pp. 217-231, Mar. 2001.

[25] K. Li, M. N. S. Swamy, and M. O. Ahmad, "An improved voice activity detection using higher order statistics," IEEE Trans. Speech Audio Process., vol. 13, pp. 965-974, Sep. 2005.

[26] P. P. Vaidyanathan, Multirate Systems and Filter Banks. Englewood Cliffs, NJ: Prentice-Hall, 1993.

[27] S. Haykin, Adaptive Filter Theory, Fourth ed. Upper Saddle River, NJ: Prentice-Hall, 2002.

[28] A. Spriet, S. Doclo, M. Moonen, and J. Wouters, "A unification of adaptive multi-microphone noise reduction systems," in Proc. Int. Workshop Acoust. Echo Noise Control, Sep. 2006, pp. 1-4.

[29] E. Nemer, R. Goubran, and S. Mahmoud, "SNR estimation of speech signals using subbands and forth-order statistics," IEEE Signal Process. Lett., vol. 6, no. 7, pp. 335-338, Jul. 1999.

[30] H. Q. Dam, S. Y. Low, H. H. Dam, and S. Nordholm, "Constrained beamforming with source PSD updates," in IEEE Proc. Int. Conf. Acoust., Speech, Signal Process., May 2004, vol. 4, pp. 93-96.

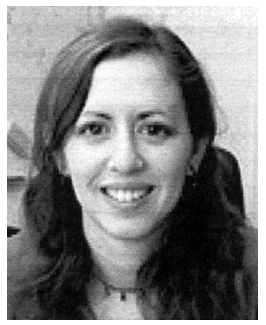

Zohra Yermeche (M'01) received the Dipl.Eng. degree in electrical engineering from the National Engineering School of Tunis, Tunis, Tunisia, in 2000. She is currently pursuing the Ph.D. degree in applied signal processing at the Blekinge Institute of Technology, Ronneby, Sweden.

Her current research interests are in array signal processing for speech enhancement, echo cancellation, dereverberation and sound source localization, as well as room acoustic modeling.

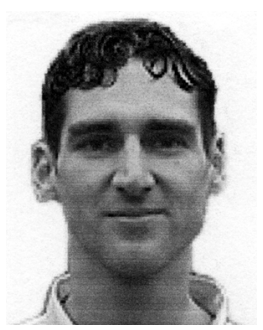

Nedelko Grbić (M'97) was born in Sweden in 1971. He received the B.Sc. degree in electrical and computer engineering from the University/College of Falun/Borlänge, Sweden, in 1993 and the M.Sc. degree in electrical engineering and the Ph.D. degree in applied signal processing from the Blekinge Institute of Technology, Ronneby, Sweden, in 1997 and 2001, respectively

He was appointed an Associate Professor in 2006 at the Blekinge Institute of Technology. His research interests include array techniques in the field of speech enhancement, adaptive beamforming, blind equalization, blind signal separation, and blind speech extraction in various applications such as binaural hearing aids, hands-free speech communication, conference telephony, and underwater acoustics.

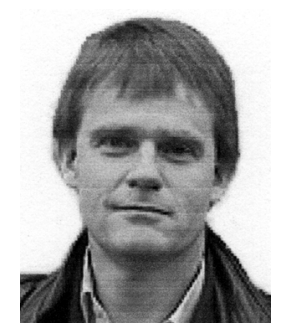

Ingvar Claesson (M'91) received the M.Sc. and Ph.D. degrees in electrical engineering from the University of Lund, Lund, Sweden, in 1980 and 1986, respectively.

Since 1990, he has been busy building a Telecommunication and Signal Processing Department at the new Blekinge Institute of Technology, Ronneby, Sweden, where he is Head of Research and holds the chair in Applied Signal Processing. He is also Dean at the University. His interests are in acoustic signal processing, filter design, adaptive filtering, and noise cancellation. He is the holder of more than 20 patents. 